\title{
Focusing light into turbid media: time- reversed ultrasonically encoded (TRUE) focusing
}

Honglin Liu, Xiao Xu, Lihong V. Wang

Honglin Liu, Xiao Xu, Lihong V. Wang, "Focusing light into turbid media: time-reversed ultrasonically encoded (TRUE) focusing," Proc. SPIE 7899, Photons Plus Ultrasound: Imaging and Sensing 2011, 78991A (18 February 2011); doi: $10.1117 / 12.876956$

SPIE. Event: SPIE BiOS, 2011, San Francisco, California, United States 


\title{
Focusing light into turbid media: time-reversed ultrasonically encoded (TRUE) focusing
}

\author{
Honglin Liu, Xiao Xu and Lihong V. Wang* \\ Optical Imaging Laboratory, Department of Biomedical Engineering, \\ Washington University in St. Louis, St. Louis, Missouri 63130, USA
}

\begin{abstract}
In turbid media such as biological tissues, light undergoes multiple scattering. Consequently, it is not possible to focus light at depths beyond one transport mean free path in such media. To break through this limit, we proposed and experimentally demonstrated a novel technique, based on ultrasonic encoding of diffused laser light and optical time reversal, which effectively focuses light into a turbid medium ${ }^{1}$. In the experimental implementation of the Time-Reversed Ultrasonically Encoded (TRUE) optical focusing, a turbid medium was illuminated by a laser beam with a long coherence length. The incident light was multiply scattered inside the medium and ultrasonically encoded within the ultrasonic focal zone. The wavefront of the ultrasonically encoded light was then time reversed by a Phase Conjugate Mirror (PCM) outside the medium. The time-reversed (or phase conjugated) optical wavefront traced back the trajectories of the ultrasonically encoded diffused light, and converged to the ultrasonic focal zone. With a commercially available photorefractive crystal as the PCM, the main approaches for increasing focusing depth are to improve the efficiencies of ultrasonic encoding and time reversal. Our recent experiments showed that light can be focused into a 5-mm thick tissue-mimicking phantom (optical thickness $=50$, i.e., geometric thickness $=50$ mean free paths) with a dynamically adjustable focus. The TRUE optical focusing opens a door to focusing light into turbid media or manipulating light-matter interactions.
\end{abstract}

Key Words: Ultrasound-modulated optical imaging, optical phase conjugation, optical time reversal, scattering medium, light manipulation.

\section{Introduction}

In turbid media, multiple scattering of light prevents focusing beyond one transport mean free path. This makes it difficult to use light as an imaging probe to "see" into a turbid medium or to manipulate light for light-matter interaction inside the medium.

To overcome the above restrictions, we proposed Time-Reversed Ultrasonically Encoded (TRUE) optical focusing which can dynamically focus light into a scattering medium. This technique combines ultrasonic modulation (or encoding) of coherent laser light in a scattering medium and optical time reversal, i.e. optical phase conjugation (OPC), of monochromatic light. Theoretically, the phase variation of the light resulted from diffusing out of the desired focus through the turbid medium can be compensated by optical phase conjugation on the wavelength scale., Thus the penetration depth could reach several centimeters in scattering-dominant turbid media, such as chicken breast tissue. The TRUE optical focus is determined by the ultrasonic focus, and not limited by the numerical apertures (NA) of optical elements. Further, the ultrasonic focus, functioning as a virtual source of the modulated light, is scalable with ultrasonic frequency and transducer aperture size, as well as adjustable to a desired spatial point. In short, TRUE optical focusing can non-invasively focus light to an arbitrary spot predefined by ultrasonic focus inside a turbid medium.

For a good understanding of TRUE optical focusing, a brief review of time reversal (TR) and ultrasoundmodulated optical imaging (UOI) is in order. Time reversal, based on the reciprocity and reversibility of wave propagation, has proven useful in reversing acoustic ${ }^{2}$ and electromagnetic ${ }^{3}$ wavefronts in applications ranging from focusing to imaging and telecommunication. Its equivalent in monochromatic light, OPC, has been widely applied for aberration correction ${ }^{4}$, optical resonators ${ }^{5}$ and high-resolution imaging . $^{6}$

*lhwang@seas.wustl.edu

Photons Plus Ultrasound: Imaging and Sensing 2011, edited by Alexander A. Oraevsky, Lihong V. Wang, Proc. of SPIE Vol. 7899, 78991A · C 2011 SPIE · CCC code: 1605-7422/11/\$18 · doi: 10.1117/12.876956 
Recently, this technique has achieved focusing through thin chicken breast tissue, which proves the validity of OPC in biological tissue.

Ultrasound-modulated optical imaging (UOI) is developed to achieve ultrasonic resolution at deep penetration in biological tissue despite of multiple light scattering, in which focused ultrasound is applied to locally encode the diffused light in the turbid media, and the encoded light is detected to retrieve the local optical information. The ultrasound-light interaction mechanisms and the various detection technologies of UOI have been widely investigated ${ }^{8-13}$. Chicken breast tissue up to $5 \mathrm{~cm}$ thickness has been imaged with millimeter resolution ${ }^{12}$.

Time reversing the ultrasonically encoded diffused light to trace the trajectories back to the ultrasonic focus - the concept of TRUE optical focusing - is achieved by combining the above two techniques. The validated experimental implementation offers a new route to manipulate light in turbid media and brings a fundamental tool for understanding and exploring the turbid world ${ }^{1}$.

\section{Methods}

\subsection{Experimental setup}

TRUE optical focusing consists of two steps. First, part of the diffused light inside a turbid medium was encoded in the ultrasonic focal zone by a running ultrasound wave (shown in Fig. 1). The modulated light $S$ interfered with a reference beam $R$ to form a static hologram recorded in a photorefractive crystal (PRC). Second, a conjugate reference beam $R^{*}$ illuminated the PRC to generate a phase conjugate wavefront $S^{*}$ and wash out the hologram simultaneously. The PRC functioned as a phase conjugate mirror. The conjugated light $S^{*}$ traced the incident trajectories back to the ultrasonic focal zone. The detailed experimental setup is shown below.

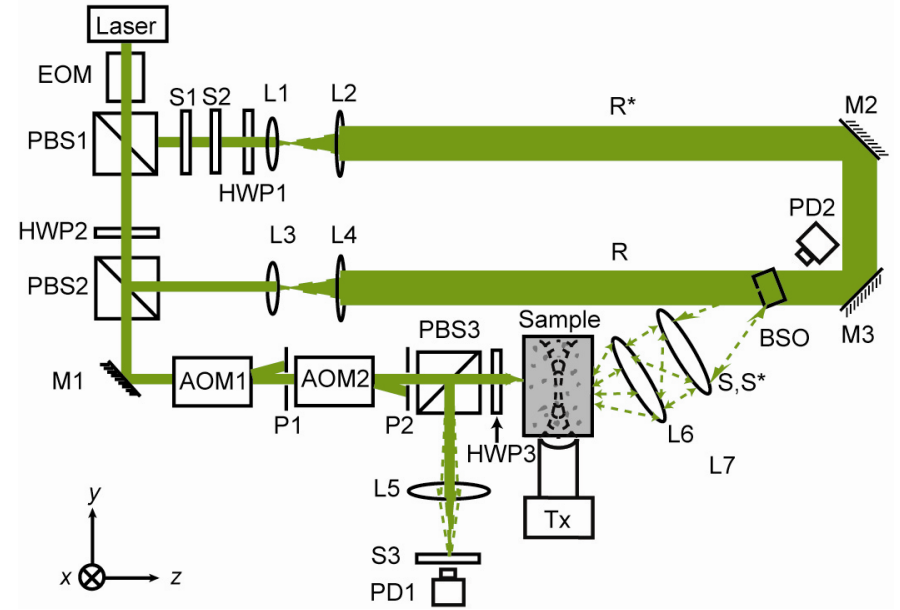

Fig. 1. Schematic of the experimental setup for TRUE optical focusing. EOM, electro-optic modulator; HWP $i$, $i$ th half-wave plate; PBS $i$, $i$ th polarizing beam splitter; $i$, $i$ th shutter; Mi, $i$ th mirror; $\mathrm{AOM} i$, $i$ th acousto-optic modulator; $\mathrm{L} i$, $i$ th lens; PD $i$, $i$ th photodiode; $\mathrm{P} i$, $i$ th iris, $\mathrm{R}$, reference beam; $R^{*}$, conjugated reference beam; $S$, modulated signal light; $S^{*}$, time-reversed signal light; $\mathrm{BSO}$, a $10 \times 10 \times 5 \mathrm{~mm}^{3} \mathrm{Bi}_{12} \mathrm{SiO}_{20}$ photorefractive crystal; Tx, an ultrasonic transducer. Coordinates: $x=$ sample scanning axis, $y=$ acoustical axis, and $z=$ optical axis. Laser output was $1.8 \mathrm{~W}$ in TRUE imaging experiments.

An incident $\mathrm{CW}$ laser (Coherent Verdi $\mathrm{V}-5, \lambda=532 \mathrm{~nm}$ ) propagated through an electro-optic modulator (EOM, Conoptics M350-50), and its polarization was switched between horizontal and vertical by different voltages applied on the EOM. The time-reversal procedure was accomplished by recording and reading of a 
hologram. In recording, the laser was switched to vertical polarization to transmit through PBS1. The transmitted light was split into two writing beams by PBS2 with a ratio adjusted by a half-wave plate HWP2. The transmitted sample beam $S$ had vertical polarization, while the reflected reference beam $R$ had horizontal polarization. The sample beam $S$ was transmitted through two acousto-optic modulators (AOM1 and AOM2, IntraAction AOM-802AF1) arranged in series to tune its optical frequency to $f_{S}=f_{0}-f_{a}$, where $f_{0}$ was the laser frequency and $f_{a}$ was the frequency shift due to the two AOMs. In a turbid sample, a focused ultrasonic wave of the same frequency $f_{a}$, launched by a focused transducer (Panametrics, A381S 3.5 MHz or A302S 1MHz), propagated in the medium and encoded the diffused light within the focal zone. The transducer was driven by a function generator (Agilent 33250A) and a 50dB gain RF power amplifier (ENI 240L). The pressure at the focus was $0.70 \mathrm{MPa}$ for the $3.5 \mathrm{MHz}$ transducer and $0.33 \mathrm{MPa}$ for the $1 \mathrm{MHz}$ transducer. The encoded light could be regarded as emanating from a virtual source overlapping with the ultrasonic focus with a frequency shift of $f_{a}$. The frequency shift resulted in two fundamental sidebands $S\left(f_{+}\right)$and $S\left(f_{-}\right)$at $f_{+}=f_{0}$ and $f_{-}=f_{0}-2 f_{a}$, respectively. Only the component $S\left(f_{+}\right)$could interfere with the reference beam $R$ to form a static hologram, which was recorded on a $10 \times 10 \times 5 \mathrm{~mm}^{3}$ BSO crystal. A high voltage AC square wave, generated by a function generator (Stanford Research System DS345) and a high voltage amplifier (TREK 609E-6), was applied on the crystal to enhance the hologram recording efficiency. In the subsequent readout, the laser was switched to horizontal polarization by the EOM and reflected by PBS1 to form the reference conjugate beam $R^{*}$. The hologram was read by beam $R^{*}$ to generate an optical time-reversed copy of $S\left(f_{+}\right)$, denoted as $S^{*}\left(f_{+}\right)$. Because of the reversibility, $S^{*}\left(f_{+}\right)$traced the trajectories of $S\left(f_{+}\right)$back to the ultrasonic focus, thereby achieved optical focusing into the scattering medium. The half-wave plate HWP1 was used to rotate the polarization of $R^{*}$ to compensate for the optical activity of the BSO. Ultrasonic emission was synchronized with the entire $200 \mathrm{~ms}$ duration of hologram recording and reading.

In the reading procedure, the transmitted $S^{*}\left(f_{+}\right)$through the sample was relayed by a lens $L 5$ onto a photodiode (PD1) as the TRUE signal. To optimize the detected TRUE signal, the ultrasonic focus was aligned to overlap the center of the diffused light profile in the sample, and HWP3 in combination with PBS3 maximize the reflection of $\mathrm{S}^{*}$ onto PD1. [[In the focal zone $S^{*}\left(f_{+}\right)$was ultrasonically modulated again, resulting in three components $S^{*}\left(f_{+}\right), S^{*}\left(f_{+}+f_{a}\right)$ and $S^{*}\left(f_{+}-f_{a}\right)$ (of frequencies $f_{0}, f_{0}+f_{a}$ and $f_{0}-f_{a}$, respectively). As a phase conjugate copy of $S\left(f_{S}\right), S^{*}\left(f_{+}-f_{a}\right)$ traced back the trajectory of $S\left(f_{S}\right)$ and exited as a coherent beam. For the components $S^{*}\left(f_{+}\right)$and $S^{*}\left(f_{+}+f_{a}\right)$, the relative phase deviation from $S^{*}\left(f_{+}-f_{a}\right)$ was much less than unity ${ }^{12}$, so that the corresponding exiting wavefront was coherent with only small phase fluctuation. All the three components contributed to the final TRUE signal.

\subsection{TRUE signal analysis}

The response time of a PRC crystal for a stable hologram is a monotonic decreasing function of recording light intensity ${ }^{14}$. Estimated from two-wave mixing experiment, the response time was about 40 milliseconds due to the low intensity of the collected modulated light in our experiment. In fact, to wipe out ambient noise, e.g., air disturbance, the recording time was set at 190 milliseconds. In the reading procedure, the intense illumination on the BSO crystal read out the stored hologram and simultaneously redistributed the trapped charge carriers in a flash. The acquired TRUE signal waveform is shown in Fig. 2, where the reading window was set at 8 milliseconds. The noise mainly came from the spatial noise in the hologram and shot noise, both of which can be reduced by averaging. 


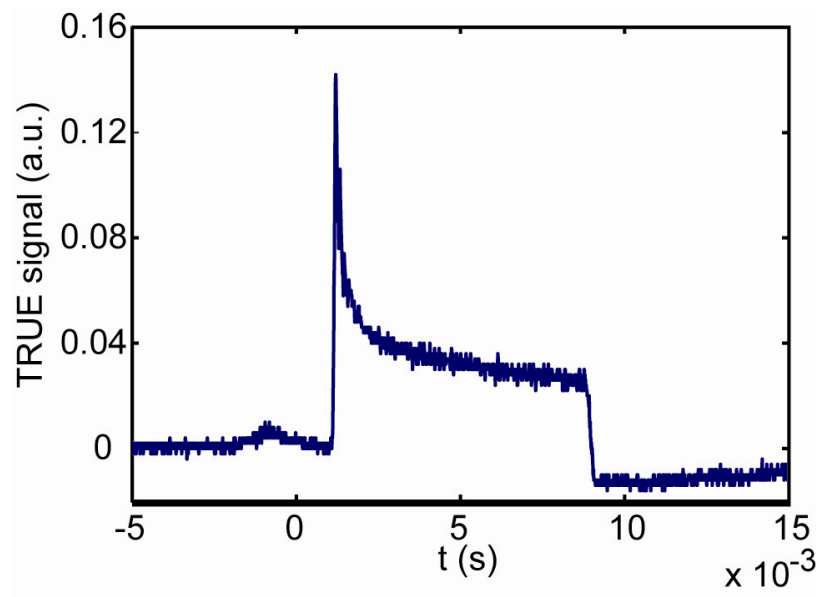

Fig. 2. Waveform of TRUE signal. Because of the finite response time of the shutter, the actual window for the photodiode was less than 8 milliseconds. The peak is the diffraction caused by the volume hologram in the BSO crystal (the TRUE signal), and the subsequent plateau is the background diffraction caused by defects and the re-distributed hologram in the BSO crystal.

Compared with the exponential decay of ballistic light, the TRUE signal, originating mainly from diffused light, decreased at a much smaller rate, as shown in Fig. 3. Obviously TRUE optical focusing has the advantage in delivering energy to targets in multiply scattering environments.

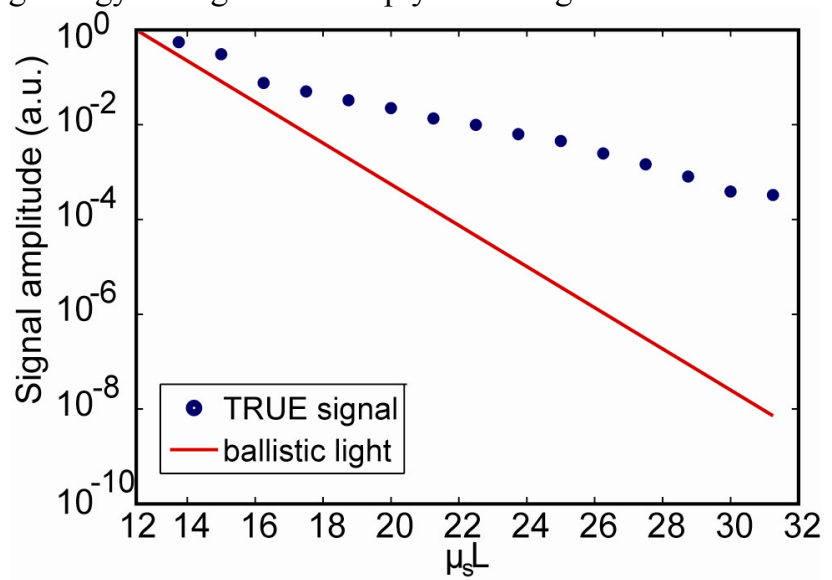

Fig. 3. Comparison of normalized TRUE signal and ballistic light at different optical thicknesses $\mu_{\mathrm{s}} \mathrm{L}$. The TRUE signal decreases at a much smaller rate, while the ballistic light decreases as a negative exponential function of optical thickness.

\section{Results}

TRUE optical focusing has been validated by imaging experiments with a tissue-mimicking scattering sample with an optical thickness $\mu_{\mathrm{s}} \mathrm{L}=25^{1}$. By updating the system with an EOM to switch light between writing and reading for higher laser utilization efficiency, we could focus light into thicker turbid media. Two samples made from porcine gelatin, water, and Intralipid were used in experiment. One was $7.5 \mathrm{~mm}$ thick with a scattering coefficient $\mu_{\mathrm{s}}=5 \mathrm{~mm}^{-1}$ and an optical thickness of 37.5. The other one was $5 \mathrm{~mm}$ thick with $\mu_{\mathrm{s}}=10 \mathrm{~mm}^{-1}$ and an optical thickness of 50 . For the first sample, by scanning along the $\mathrm{x}$-axis we obtained TRUE and UOT images using the $3.5 \mathrm{MHz}$ transducer (focal length $=38 \mathrm{~mm}$, aperture diameter = $19.05 \mathrm{~mm}$, and focal width $=0.87 \mathrm{~mm}$ ). We also obtained a TRUE image with the $1 \mathrm{MHz}$ transducer (focal length $=41.4 \mathrm{~mm}$, aperture diameter $=25.4 \mathrm{~mm}$, and focal width $=2.49 \mathrm{~mm}$ ) for comparison. The results are shown in Fig.4. 

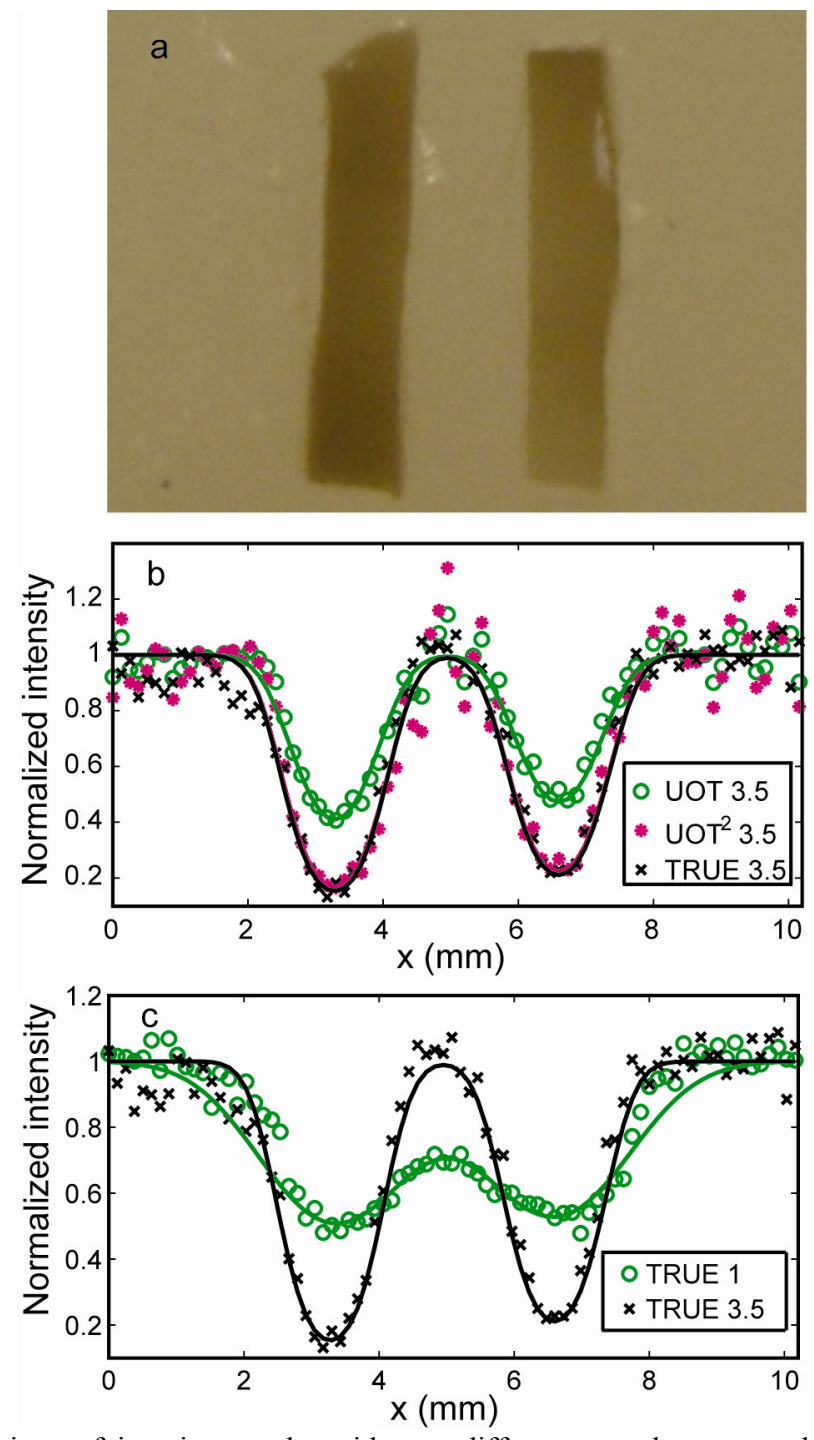

Fig. 4. Comparison of imaging results with two different transducers. a, photograph of the imaged sample dissected at the middle plane which contains two absorbing objects (Obj1 and Obj2). The object dimensions were $x=1.5 \mathrm{~mm}, y=6.0 \mathrm{~mm}$, and $z=0.8 \mathrm{~mm}$, while the full dimensions of the sample were $x=y=60 \mathrm{~mm}$ and $z=7.5 \mathrm{~mm}$. b, comparison of the normalized UOT and TRUE images of the sample using the $3.5 \mathrm{MHz}$ transducer, consistent with the square law: the TRUE signal is proportional to the square of the UOT signal $\left(\mathrm{UOT}^{2}\right)$. c, comparison of the normalized TRUE images of the sample with $3.5 \mathrm{MHz}$ and $1 \mathrm{MHz}$ transducers. The contrast of the TRUE image was $73 \%$ with the $3.5 \mathrm{MHz}$ transducer, and only $16 \%$ for the $1 \mathrm{MHz}$ transducer. In $\mathbf{b}$ and $\mathbf{c}$, the discrete symbols represent experimental data while the solid curves represent Gaussian fits.

Fig. 4a shows a photograph of the two absorption objects with dimensions of $1.5 \times 6.0 \times 0.8 \mathrm{~mm}^{3}$, which were made by adding black India ink in the phantom resulting in an absorption coefficient $\mu_{\mathrm{a}}=1.17 \mathrm{~mm}^{-1}$ at 532 $\mathrm{nm}$. As shown in Fig .4b, both of the TRUE and UOT images encoded by the $3.5 \mathrm{MHz}$ ultrasonic wave accurately distinguished the two objects from the background. The TRUE image agrees well with the UOT ${ }^{2}$ image, in accord with the previously established square law ${ }^{1}$. The resolutions of the TRUE and UOT images - defined by the full widths at half maxima (FWHM's) of the approximated Gaussian profiles of the $3.5 \mathrm{MHz}$ transducer - were $0.63 \mathrm{~mm}$ and $0.88 \mathrm{~mm}$, respectively. Hence, light was demonstrated to focus into a scattering sample of an optical thickness 37.5. Fig. 4c compares two TRUE images of the same sample with the $3.5 \mathrm{MHz}$ (shown in black crosses and solid line) and $1 \mathrm{MHz}$ (shown in green circles and 
solid line) transducers. The $1 \mathrm{MHz}$ TRUE image had a resolution of $1.60 \mathrm{~mm}(2.49 / 1.60=1.5)$ and poorer contrast, since the light was focused to a bigger spot as determined by the $1 \mathrm{MHz}$ transducer's focus.

A main motivation of TRUE optical focusing is to see deeply into thick scattering media and to manipulate light-matter interaction in such turbid media. Taking advantage of the higher encoding efficiency and a larger focal volume of low frequency ultrasonic wave, we focused light into a $5 \mathrm{~mm}$ thick tissue mimicking sample with a scattering coefficient $\mu_{\mathrm{s}}=10 \mathrm{~mm}^{-1}$. Fig. 5 shows the TRUE imaging results of the sample using the $1 \mathrm{MHz}$ transducer. Fig. 5a is a photograph of the sample's middle plane, which contains three absorbing objects with the same scattering coefficient as the background and higher absorption coefficient of $\mu_{\mathrm{a}}=1.17 \mathrm{~mm}^{-1}$. Fig. $5 \mathrm{~b}$ shows the experimental data and the corresponding fitting curve obtained by convolution of the object profile with the approximated Gaussian profile of the $1 \mathrm{MHz}$ ultrasonic focus. The imaging resolution was $1.74 \mathrm{~mm}$, which has a 1: $\sqrt{2}$ ratio compared with the $1 \mathrm{MHz}$ transducer's focal width of $2.49 \mathrm{~mm}$.

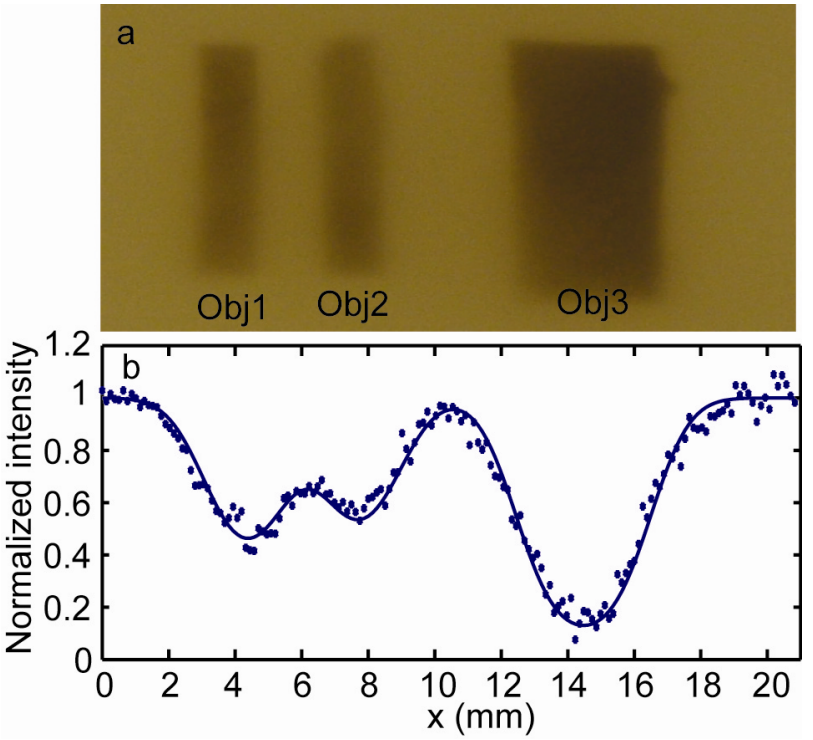

Fig. 5. TRUE image of a $5 \mathrm{~mm}$ thick phantom sample with the $1 \mathrm{MHz}$ transducer. a, photograph of the $5 \mathrm{~mm}$ thick sample dissected at the middle plane containing three absorbing objects (Obj1, Obj2 and Obj3). The widths of the three objects were $x=1.7 \mathrm{~mm}, 1.5 \mathrm{~mm}$ and $3.3 \mathrm{~mm}$, respectively; and their lengths and thicknesses were $y=5.2 \mathrm{~mm}$, and $z=0.8 \mathrm{~mm}$. The full dimensions of the sample were $x=y=60 \mathrm{~mm}$ and $z=5 \mathrm{~mm}$. b, a TRUE image of the sample using the $1 \mathrm{MHz}$ transducer. The dots represent experimental data and the solid curve represents Gaussian fit.

The distances between the first two objects are smaller than the ultrasonic focal width. Their edges overlap, resulting in lower contrast. However, the third object is sufficiently far away from the first two objects to be clearly distinguished from the background. The consistence of the resolution and the ultrasonic focus verifies that light was focused to the $1 \mathrm{MHz}$ transducer focal zone inside the sample.

\section{Discussion}

In a turbid medium, a multiple-scattering trajectory can be considered as a photon propagation channel. In this view, TRUE optical focusing is realized by sending photons through the optical channels marked in the writing procedure back to the original virtual source. To ensure that photons be transported to the focus, the marked channels should be stable during time reversal, which means that the medium de-correlation time should be longer than the duration of TRUE optical focusing. The de-correlation time is influenced by a number of factors. In addition to the inherent Brownian motion and physiological movement of biological tissue, sample oscillation caused by high pressure ultrasound and particle motion enhanced by optical and acoustic energy deposition will also distort the marked channels, which will gradually lead to the collapse of time reversal. This behavior has been demonstrated by the diminishing TRUE signal upon certain 
thresholds of ultrasound pressure and laser output power. Hence, using dynamic holographic material with a large sensitive area, instead of increasing ultrasonic pressure and laser output power, will play a more important role in focusing into thicker turbid media. Real or near-real time focusing with a faster photorefractive material is also a goal.

Theoretically, in order to faithfully time reverse a light propagation process, the wavefront within the full $4 \pi$ solid angle should be recorded and phase conjugated. The finite collecting aperture of a photorefractive crystal results in imperfect wavefront replication. In conventional aberration correction, the fidelity of OPC is characterized by the smallest transverse scale of aberration that can be compensated, which is of the order $L / a$, where $L$ is distance of a phase conjugate mirror (PCM) (a BSO crystal in our experiment) away from the exiting plane of diffused light, and $a$ is the aperture size of the $\mathrm{PCM}^{15}$. The correctable scale ranges up to $L / a$. However, because of multiple scattering, this limitation on the transverse scale has little restriction on TRUE optical focus. Multiple scattering can dramatically broaden the light angular distribution $^{16,17}$, and consequently, compensate for the limited angular width of a PCM system. In principle the resolution of TRUE optical focusing is not limited by the finite aperture of a PCM system.

In this work it took more than a hundred milliseconds to form a stable hologram for the weak interference pattern of the modulated light, whereas the erasing time, the width of the peak in Fig.2, was less than 1 millisecond because the reference conjugated beam washed out the hologram instantly under intense illumination. Higher reading power can increase the amplitude while compressing the width of the peak, i.e., high power short pulse light in the focus can be achieved, which provides a potential new tool to study light excited phenomena, such as fluorescence and the photoacoustic effect. In order to avoid hologram erasure to deliver more energy to the focus in the reading procedure, the volume hologram can be fixed by heating the crystal to hundreds of degrees or by applying an electric field to the crystal. Once the hologram is fixed, in playing back, numerous photons can be focused into turbid media to interact with and even burn particles, cells, and clusters within the ultrasonic focus. Besides hologram fixing, non-destructive read-out of holograms can also be realized by the two-photon storage technique ${ }^{14}$.

\section{Conclusion}

In summary, using an updated implementation of TRUE optical focusing, we have focused light into a 5 $\mathrm{mm}$ thick tissue-mimicking scattering medium. We also demonstrated that the focal spot is determined by the ultrasound wave, and it is scalable with the ultrasonic frequency and transducer aperture. TRUE optical focusing offers a valuable tool for light manipulation inside turbid media which helps us view and understand the turbid world.

\section{Acknowledgement}

This research is funded in part by the National Institutes of Health grants R01 EB000712 and U54 CA136398. 


\section{References}

[1] X. Xu, H. Liu, and L. V. Wang, "Time-reversed ultrasonically encoded optical focusing," Nature Photon. In press (2011).

[2] M. Fink, D. Cassereau, A. Derode, C. Prada, P. Roux, M. Tanter, J-L. Thomas, and F. Wu, "Timereversed acoustics," Rep. Prog. Phys. 63, 1933-1995 (2000).

[3] G. Lerosey, J. de Rosny, A. Tourin, and M. Fink, "Focusing beyond the diffraction limit with farfield time reversal," Science 315, 1120-1122 (2007).

[4] I. V. Tomov, R. Fedosejevs, D. C. D. McKen, C. Domier, and A. A. Offenberger, "Phase conjugationand pulse-compression of KrF-laser radiation by stimulated Raman-scattering," Opt. Lett. 8, 9-11 (1983).

[5] R. A. McFarlane, and D. G. Steel, "Laser-oscillator using resonator with self-pumped phaseconjugate mirror," Opt. Lett. 8, 208-210 (1983).

[6] M. D. Levenson, "High-resolution imaging by wave-front conjugation," Opt. Lett. 5, 182-184 (1980).

[7] Z. Yaqoob. D. Psaltis. M. S. Feld, and C. Yang, "Optical phase conjugation for turbidity suppression in biological samples," Nature Photon. 2, 110-115 (2008).

[8] F. A. Marks. H. W. Tomlinson, and G. W, Brooksby, "A comprehensive approach to breast cancer detection using light: photon localization by ultrasound modulation and tissue characterization by spectral discrimination," Proc. SPIE, 1888, 500-510 (1993).

[9] L. V. Wang, "Mechanisms of ultrasonic modulation of multiply scattered coherent light: an analytic model," Phy. Rev. Lett. 87, 043903-043906 (2001).

[10] B. C. Forget. F. Ramaz, M. Atlan, J. Selb, and A. C. Boccara, "High-contrast fast Fourier transform acousto-optical tomography of phantom tissues with a frequency-chirp modulation of the ultrasound," Appl. Opt. 42, 1379-1383 (2003).

[11] S. Sakadzic and L. V. Wang, "High-resolution ultrasound-modulated optical tomography in biological tissues," Opt. Lett. 29, 2770-2772 ( 2004).

[12] X. Xu, H. L. Zhang, P. Hemmer, D. K. Qing, C. H. Kim, and L. V. Wang, "Photorefractive detection of tissue optical and mechanical properties by ultrasound modulated optical tomography," Opt. Lett. 32, 656-658 (2007).

[13] Y. Z. Li, H. L. Zhang, C. H. Kim, K. H. Wagner, P. Hemmer, and L. V. Wang, "Pulsed ultrasound-modulated optical tomography using spectral-hole burning as a narrowband spectral filter," Appl. Phys. Lett. 93, 011111-(1-3) (2008).

[14] L. Solymar, D. J. Webb, and A. Grunnet-Jepsen, "The Physics and Applications of Photorefractive Materials." (Clarendon Press, Oxford, 1996).

[15] R. Giuliano Concetto, "Applications of optical phase conjugation," Physics Today, p. 27, April 1981.

[16] I. M. Vellekoop, A. Lagendijk, and A. P. Mosk, "Exploiting disorder for perfect focusing," Nature Photon. 4, 320-322, (2010).

[17] M. Cui, E. J. McDowell, and C. Yang, "Observation of polarization-gate based reconstruction quality improvement during the process of turbidity suppression by optical phase conjugation," App. Phys. Lett. 95(12), 123702 (2009). 